Communication

\title{
A Theoretical Framework to Determine RHP Zero Dynamics in Sequential Interacting Sub-Systems
}

\author{
Anca Maxim ${ }^{1,2, *(1)}$, Riccardo Ferracuti ${ }^{3,+}$ and Clara M. Ionescu ${ }^{1,4}$ (i) \\ 1 DySC Research Group on Dynamical Systems and Control, Department of Electrical Energy, Metals, \\ Mechanical Constructions and Systems, Ghent University, Technologiepark 125, B-9052 Ghent, Belgium; \\ claramihaela.ionescu@ugent.be \\ 2 Department of Automatic Control and Applied Informatics, "Gheorghe Asachi” Technical University of Iasi, \\ Blvd. Mangeron 27, 700050 Iasi, Romania \\ 3 Independent Researcher, 20159 Milan, Italy; riccardoferra@gmail.com \\ 4 Department of Automation, Technical University of Cluj-Napoca, Memorandumului Street 28, \\ 400114 Cluj-Napoca, Romania \\ * Correspondence: anca.maxim@ac.tuiasi.ro \\ + Formerly with Department of Information Engineering, University of Padova, Via Gradenigo 6, \\ B-35131 Padova, Italy.
}

Received: 8 April 2019; Accepted: 6 May 2019; Published: 10 May 2019

\begin{abstract}
A theoretical framework for determining the dynamics of interacting sub-systems is proposed in this paper. Specifically, a systematic analysis is performed that results in an indication about whether an MP or an NMP dynamics occurs in the analyzed process during operation. The analysis stems from the physical process description and the degree of coupling between sub-systems. The presented methodology is generalized for $n$ sub-systems with sequential interaction (i.e., in which the coupling is unidirectional and occurs between consecutive sub-systems), and the outcome is useful investigation tool prior to the controller design phase. Given the generality of the approach, the theoretical framework is valid for any dynamic process with interacting sub-systems in the context of LTI systems.
\end{abstract}

Keywords: MP dynamics; NMP dynamics; multivariable process; sequential interacting sub-systems; LTI system; RHP transmission zero

\section{Introduction}

From the control engineering point of view, the closed-loop performance in systems exhibiting NMP behavior presents serious limitations in achievable bandwidth. In this context, it is advantageous to know a priori the dynamics of the system at hand, before specifying any requirements of the closed-loop performance, e.g., settling time and overshoot. Generically, the research objectives for this topic lead towards: (i) developing control strategies able to deal with the NMP challenges [1-4] and (ii) analyzing the behavior prior to the control design stage [5-7].

This paper proposes a generally valid mathematical framework to determine the system dynamics for sequential interacting sub-systems. The study case at hand is a generalization for a particular class of systems composed of several sub-systems in a train architecture. This specific characteristic appears in supply-chain management [8], water distribution networks [9,10], distillation columns [11], and other complex interactive processes [12,13]. This knowledge is also important when deciding the coupling of the sub-system in a minimal information structure for distributed control [14]. The study that is presented in this paper can be regarded as a system analysis that must be performed prior to controller design, since NMP systems have different dynamics and different responses than MP ones. 
The motivation of this work is to provide a sound analysis of the system dynamics, for this particular class of interacting sub-systems, that can be used prior to the control design. This is done without using identification techniques, if the coupling degree between sub-systems is known.

The main contribution of this paper is based on analysis made on a laboratory setup available at Ghent University and can be summarized as follows [15]:

- it delivers a generic polynomial expression to characterize the information about the presence of transmission zeros of a MIMO system, employing the Smith-McMillan decomposition method and

- it proposes a computationally-efficient method to evaluate the solution of the polynomial by means of Descartes' rule of signs.

Notation: $\mathbb{R}[s]$ is the set of polynomials with coefficients in $\mathbb{R} ; \mathbb{R}(s)$ is the set rational functions; $\mathbb{R}[s]^{m \times n}$ denotes the set of $m \times n$ matrices, whose entries are polynomials; and $a \mid b$ stands for $a$ divides into $b$.

\section{The Polynomial Form to Determine Transmission Zeros}

The textbook definition indicates that in multivariable systems, the transmission zeros are defined when the system matrix is rank deficient [16]. This rule is a basis for the remainder of this work. Let $G(s) \in \mathbb{R}[s]^{n \times n}$ be the transfer function matrix of the $n$-interacting sub-systems:

$$
G(s)=\left[\begin{array}{ccccc}
G_{1,1} & 0 & \cdots & 0 & G_{1, n} \\
G_{2,1} & G_{2,2} & 0 & \cdots & 0 \\
\vdots & \vdots & \vdots & \ddots & \vdots \\
0 & 0 & \cdots & G_{n, n-1} & G_{n, n}
\end{array}\right]
$$

where each sub-system is described by two transfer functions with three distinct time constants placed in a row in (1) (i.e., a first order and a second order transfer function, respectively). Hence, one may, without loss of generality, assume first order transfer functions on the main diagonal:

$$
G_{i, i}(s)=\frac{c_{i} \gamma_{i}}{\left(T_{3 i} s+1\right)}
$$

and second order transfer functions below the main diagonal:

$$
G_{i, i-1}(s)=\frac{c_{i}\left(1-\gamma_{i-1}\right)}{\left(T_{3 i-2} s+1\right)\left(T_{3 i-1} s+1\right)}
$$

where $i=\overline{2, n}$ and $n$ is the number of sub-systems. Note that for $i=1, G_{1,1}=c_{1} \gamma_{1} /\left(T_{1} s+1\right)$ and $G_{1, n}=c_{1}\left(1-\gamma_{n}\right) /\left(T_{2} s+1\right)\left(T_{3} s+1\right)$ are the transfer functions corresponding to the first module coupled circularly with the last module (e.g., as in recirculation loops). Each transfer function has a gain depending on two constant values determined by the physical properties of the assembly: $\gamma_{i}$ and $c_{i}, i=\overline{1, n}$. The value $0<\gamma_{i}<1$ represents the interaction coefficient, which characterizes the coupling degree among the modules. The value $c_{i}$ is a constant depending on system specification. The remaining variable $T_{i}$ is a time constant dependent on $\gamma_{i}$ and $c_{i}$.

Using the Smith-McMillan form [17], the zero polynomial can be computed from (1) as:

$$
z(s)=\prod_{i=1}^{n} \epsilon_{i}(s)
$$

where $\epsilon_{i}, i=\overline{1, n}$ are the diagonal elements of the Smith matrix $S(s)=G(s) h(s)$, with $h(s)$ a polynomial that is the LCM of the denominators of all the elements in $G(s)$. Using the property $\epsilon_{i}(s) \mid \epsilon_{i+1}(s), i=$ 
$\overline{1, n-1}$, it results that the solutions of (2) can be determined only through the evaluation of the last element:

$$
\epsilon_{n}=\frac{\Delta_{n}}{\Delta_{n-1}}
$$

where $\Delta_{n}$ is the GCD of all $n \times n$ minors of $S(s)$.

Obviously, this textbook method loses computational efficiency with the increasing number of sub-systems $n$; e.g., for $n=10$, the GCD among 100 different matrices needs to be computed. Hence, an alternative, efficient solution suitable for a general formulation, which exploits the particular structure of the transfer function matrix (1), is provided hereafter. For the case of $n$ interacting sub-systems and with the polynomial $h(s)=\left(s T_{1}+1\right)\left(s T_{2}+1\right) \cdots\left(s T_{3 n-1}+1\right)\left(s T_{3 n}+1\right)$, which collects all the distinct denominators from (1), hence the $3 n$ degree, the Smith matrix $S(s)$ has the following entries:

$$
\begin{aligned}
S_{1,1}(s) & =c_{1} \gamma_{1}\left(T_{2} s+1\right)\left(T_{3} s+1\right) \cdots\left(T_{3 n} s+1\right) \\
S_{1, n}(s) & =c_{1}\left(1-\gamma_{n}\right)\left(T_{1} s+1\right)\left(T_{4} s+1\right) \cdots\left(T_{3 n} s+1\right) \\
\vdots & \\
S_{n, n}(s) & =c_{n} \gamma_{n}\left(T_{1} s+1\right) \cdots\left(T_{3 n-1} s+1\right)
\end{aligned}
$$

The above polynomials can be classified, with respect to the degree, into two disjoint sets. Hence, $\mathcal{Q}$ contains all the polynomials of $3 n-1$ degree (i.e., $\mathcal{Q}=\left\{S_{1,1}, S_{2,2}, \cdots, S_{n, n}\right\}$ ), and $\mathcal{R}$ contains all the polynomials of $3 n-2$ degree (i.e., $\mathcal{R}=\left\{S_{1, n}, S_{2,1}, \cdots, S_{n, n-1}\right\}$ ).

Proposition 1. Let $S(s) \in \mathbb{R}[s]^{n \times n}$ be the Smith matrix, and given two disjoint sets $\mathcal{Q}$ and $\mathcal{R}$, the determinant has the following structure:

$$
\operatorname{det}(S)=\prod_{i=1}^{n} S_{i, i}+(-1)^{n+1} \prod_{j=2}^{n} S_{j, j-1} S_{1, n}
$$

where the elements $S_{i, i} \in \mathcal{Q}$ and the elements $S_{i, j} \in \mathcal{R}$ with $i \neq j$.

The proof for Proposition 1 is determined straightforwardly through induction, starting from $n=2$.

Proposition 2. Let $S(s) \in \mathbb{R}[s]^{n \times n}$ be the Smith matrix, and given two disjoint sets $\mathcal{Q}$ and $\mathcal{R}$, the determinant of the minor $n-1$ of $S$ can have three mutually exclusive structures:

$$
\operatorname{det}\left([S]_{i, j}^{n-1}\right)= \begin{cases}\prod_{i=1}^{n-1} S_{i, i} & A \\ (-1)^{n} \prod_{j=2}^{n-1} S_{j, j-1} S_{1, n} & B \\ \prod_{i, j=1}^{n-1} S_{i, j} & C\end{cases}
$$

where Cases $A$ and $B$ are evaluated only with elements from sets $\mathcal{Q}$ and $\mathcal{R}$, respectively, and Case $C$ has elements from both sets.

The proof of Proposition 2 is determined by investigating the three possibilities to compute an $n-1$ minor by deleting a row $i$ and column $j$ (i.e., $i=j$ for Case $\mathrm{A}, i<j$ for $\mathrm{B}$ and $i>j$ for $\mathrm{C}$ ). 
Proposition 3. Let $G(s) \in \mathbb{R}[s]^{k \times k}$ be a transfer function matrix with poles of multiplicity $1, S(s) \in \mathbb{R}[s]^{k \times k}$ a Smith matrix associated with $G(s)$, and $k \in \mathbb{N}$, with $k>2$, then the product of $k$ different elements in $S(s)$ has the form:

$$
\prod_{i, j=1}^{k} S_{i, j}=h^{k-1}(s) p_{k}(s)
$$

where $h(s)$ is the LCM of the denominators in $G(s)$ and $p_{k}(s)$ is a polynomial that depends on the elements $S_{i, j}$.

Proposition 3 allows one to separate the product of $k$ different non-zero elements of the matrix $S(s)$ into two terms:

- $h(s)$, a fix polynomial whose roots do not depend on the product's elements and

- $\quad p_{k}(s)$, a polynomial whose roots depend on the product's elements.

As such, using Proposition 3 and (5), the following results for $\Delta_{n-1}$ and $\Delta_{n}$ are obtained:

$$
\begin{aligned}
\Delta_{n-1}(s)= & G C D\left\{[S]_{i, j}^{n-1}(s)\right\}=G C D\left\{\operatorname{det}\left([S]_{i, j}^{n-1}(s)\right)\right\} \\
= & G C D\left\{h^{n-2}(s) p_{i, j}(s)\right\}=h^{n-2}(s) \\
& \forall i, j=\overline{1, n} . \\
\Delta_{n}(s)= & G C D\left\{[S]_{i, j}^{n}(s)\right\}=G C D\{S(s)\}=\operatorname{det}(S(s)) \\
= & \prod_{i=1}^{n} S_{i, i}(s)+(-1)^{n+1} \prod_{j=2}^{n} S_{j, j-1}(s) S_{1, n}(s) .
\end{aligned}
$$

The equality in (6) holds for the polynomial $h(s)$, as it is independent of $i$ and $j$. After some algebraic manipulation (7) can be written for $\Delta_{n}(s)$ as:

$$
\begin{aligned}
& \prod_{i=1}^{n} c_{i} h^{n-1}(s)\left[\prod_{i=1}^{n} \gamma_{i}\left(s T_{2}+1\right) \cdots\left(s T_{3 n-1}+1\right)+\right. \\
& \left.\quad+(-1)^{n+1} \prod_{i=1}^{n}\left(1-\gamma_{i}\right)\left(s T_{1}+1\right) \cdots\left(s T_{3 n}+1\right)\right]
\end{aligned}
$$

Having (8) and (6), one can compute (3), and after the division of $\epsilon_{n}$ by $h(s)$, accordingly with the Smith-McMillan form, the final form for the transmission zero polynomial is:

$$
\begin{gathered}
\epsilon_{n}(s)=\prod_{i=1}^{n} c_{i}[\underbrace{\left.\prod_{i=1}^{n} \gamma_{i}\left(s T_{2}+1\right)\right) \cdots\left(s T_{3 n-1}+1\right)}_{\epsilon_{A}(s)}+ \\
+(-1)^{n+1} \underbrace{\prod_{i=1}^{n}\left(1-\gamma_{i}\right)\left(s T_{1}+1\right) \cdots\left(s T_{3 n}+1\right)}_{\epsilon_{B}(s)}]
\end{gathered}
$$

where $\epsilon_{A}(s)$ is a $2 n$ degree polynomial and $\epsilon_{B}(s)$ is a $n$ degree polynomial, both with all the coefficients strictly positive (Under the condition that $\gamma_{i}$ belongs to: $0<\gamma_{i}<1 \forall i=\overline{1, n}$ ), defined as follows:

$$
\begin{aligned}
& \epsilon_{A}(s)=\alpha_{2 n} s^{2 n}+\cdots+\alpha_{n} s^{n}+\cdots+\alpha_{1} s+\alpha_{0} \\
& \epsilon_{B}(s)=\beta_{n} s^{n}+\cdots+\beta_{1} s+\beta_{0}
\end{aligned}
$$

\section{Evaluation of the Transmission Zero Polynomial}

The roots of the zero polynomial depend on the number of modules $n$ present in the system (i.e., whether $n$ takes odd or even values, it influences the sign of the second term in (9)). We employ 
Descartes' rule of signs to analyze the roots of (9) (i.e., depending on the number of sign differences between consecutive non-zero coefficients, the number of positive roots in a polynomial can be evaluated). By definition, an NMP system has at least one positive transmission zero. Consequently, one only needs to determine when the first change of sign appears in the roots to conclude upon the existence of such a positive zero.

Definition 1. Let $\mathcal{C}$ be the set of all possible denominators in the off-diagonal of $G(s)$, thus containing the roots of $\epsilon_{A}(s)$. Let $\mathcal{D}$ be the set of all possible denominators in the main-diagonal of $G(s)$, such that it contains the roots of $\epsilon_{B}(s)$.

To determine the sign of the polynomial $\epsilon_{n}(s)$, only the coefficients in the range $[0-n]$ are necessary, while in (9), beyond the degree $n$, they are only the components from (10). Hereafter, a relation between the coefficients of (10) and (11) is given.

Proposition 4. Let $\epsilon_{A}(s)$ and $\epsilon_{B}(s)$ be two polynomials such that:

(1) $\operatorname{deg}\left[\epsilon_{A}(s)\right]=2 \operatorname{deg}\left[\epsilon_{B}(s)\right]$

(2) $T_{i} T_{j}>T_{k} \forall\left(T_{i}, T_{j}\right) \in \mathcal{C}, \forall\left(T_{k}\right) \in \mathcal{D}$.

then: $\alpha_{0}>\beta_{0} \Rightarrow \alpha_{k}>\beta_{k}, \forall k=\overline{1, n}$.

The proof is given in Appendix A.

Remark 1. The first $(2 n-n)$ coefficients of $\epsilon_{n}(s)$ are always positive. If at least one of the remaining coefficients is negative, then the coefficient of degree zero is guaranteed to be negative.

Therefore, in (9), when $n$ takes odd values, all coefficients are strictly positive. Applying Descartes rule of signs, it follows that all the roots are negative (i.e., there are not any sign changes between consecutive coefficients). On the other hand, when $n$ takes even values, according to Proposition 3 , it suffices to check the sign of the first coefficient, in order to determine whether the system has either MP or NMP dynamics.

\section{Analysis of the Transmission Zero Polynomial}

Following the proposed method, the analysis of $n$-interconnected sub-systems depends on the sign of the zero degree coefficient from the transmission zero polynomial. Replacing $s=0$ in (9) gives:

$$
\zeta_{0}=\prod_{i=1}^{n} c_{i}\left(\prod_{i=1}^{n} \gamma_{i}-\prod_{i=1}^{n}\left(1-\gamma_{i}\right)\right)
$$

Defining with $\Gamma=\left\{\gamma_{1}, \gamma_{2}, \cdots, \gamma_{n}\right\}$ the set that contains all possible values of $\gamma$ and using the binomial coefficient, (12) can be written as:

$$
\begin{aligned}
\zeta_{0} & =\prod_{i=1}^{n} c_{i}\left(-1+\sum_{i=1}^{n-1}(-1)^{i}\left(\begin{array}{c}
n \\
i
\end{array}\right) \Gamma\right)= \\
& =\prod_{i=1}^{n} c_{i}(-1+\underbrace{\gamma_{1}+\gamma_{2}+\cdots+\gamma_{n}}_{n} \underbrace{-\gamma_{1} \gamma_{2}-\gamma_{2} \gamma_{2}-\cdots}_{\frac{n(n-1)}{2}})
\end{aligned}
$$


Next, the expected value of the set $\Gamma$ (i.e., $\hat{\gamma}=\mathbb{E}[\Gamma]=\frac{1}{n} \sum_{i=1}^{n} \gamma_{i}$ ) is used to perform a variable change in (13), leading to:

$$
\begin{aligned}
\zeta_{0}(\hat{\gamma}) & =n \hat{\gamma}^{n-1}-\frac{n(n-1)}{2} \hat{\gamma}^{n-2}+\cdots \\
& -\frac{n(n-1)}{2} \hat{\gamma}^{2}+n \hat{\gamma}-1 \\
& =\hat{\gamma}^{n}-(\hat{\gamma}-1)^{n}
\end{aligned}
$$

Proposition 5. Let $n \in \mathbb{N}$ be an even number and $\hat{\gamma} \in \mathbb{R}$ be defined in the interval $I=(0,1)$, then:

$$
\zeta_{0}(\hat{\gamma})=\hat{\gamma}^{n}-(\hat{\gamma}-1)^{n} \quad \forall n=2,4, \cdots
$$

has only one real solution for $\hat{\gamma}=\frac{1}{2}$.

The proof is given in Appendix A.

For a better understanding, a case with $n=4$ is further exemplified. The set of all possible values of $\gamma$ is $\Gamma=\left\{\gamma_{1}, \gamma_{2}, \gamma_{3}, \gamma_{4}\right\}$, while the coefficient $\zeta_{0}$ from (13) and the polynomial $\zeta_{0}(\hat{\gamma})$ given in (14) are:

$$
\begin{gathered}
\zeta_{0}=\prod_{i=1}^{n} c_{i}(-1+\underbrace{\gamma_{1}+\cdots+\gamma_{4}}_{4}-\underbrace{\gamma_{1} \gamma_{2}-\gamma_{1} \gamma_{3}-\cdots-\gamma_{3} \gamma_{4}}_{6}+\underbrace{\gamma_{1} \gamma_{2} \gamma_{3}+\cdots+\gamma_{4} \gamma_{1} \gamma_{2}}_{4}), \\
\zeta_{0}(\hat{\gamma})=\prod_{i=1}^{n} c_{i}\left(4 \hat{\gamma}^{3}-6 \hat{\gamma}^{2}+4 \hat{\gamma}-1\right)
\end{gathered}
$$

The solutions of (16) are:

$$
\zeta_{0}(\hat{\gamma})=0 \Rightarrow\left\{\begin{array}{l}
\hat{\gamma}_{1}^{*}=\frac{1}{2} \\
\hat{\gamma}_{2}^{*}=\frac{1}{2}+\frac{1}{2} i \\
\hat{\gamma}_{3}^{*}=\frac{1}{2}-\frac{1}{2} i
\end{array}\right.
$$

Since $\gamma \in \mathcal{R}$, we are only interested in the real solution. Therefore, all $\gamma_{i}, i=\overline{1,4}$ combinations with $\hat{\gamma}=\frac{1}{2}$ will bring the coefficient $\zeta_{0}$ to zero. If we consider $\Gamma=\{0.3,0.6,0.4,0.7\}$, the expected value is $\hat{\gamma}=\frac{1}{2}$, and substituting the values in (15), we have:

$$
\zeta_{0}=\prod_{i=1}^{n} c_{i}(-1+2-1.45+0.45)=0 .
$$

For the particular study case proposed in this work, when there is an even number of modules, the interacting system has MP behavior, if and only if the average value of the interaction coefficients: $\mathbb{E}\left[\gamma_{i}\right]<0.5$. Otherwise, the process has NMP behavior. If the average value is $\mathbb{E}\left[\gamma_{i}\right]=0.5$, a transmission zero appears in the origin. The above analysis stands for all systems composed of an even number of sub-systems. However, a process with an odd number of sub-systems has always MP dynamics, because all the roots of the polynomial (9) are negative.

Moreover, the proposed method was successfully applied on the sextuple tank process available at Ghent University, consisting of three coupled sub-systems, which always has an MP dynamics. After that, the interacting process was controlled with a distributed predictive control technique, with the results available in [14]. 


\section{Conclusions}

In this work, the Smith-McMillan form is used to compute the polynomial that contains the transmission zeros of a general process with $n$ interacting sub-systems efficiently. The method does not require identification to determine if performance limitations exist from the presence of RHP transmission zeros, but only interaction degree information. Starting from a formal mathematical formulation, a novel result was obtained, in which the NMP behavior can be identified by simply counting the interacting sub-systems. When the system is composed of an even number of sub-systems, then a simple condition based on the average value of the interaction degree parameters must be checked. Furthermore, the study also shows that this finding further simplifies when an odd number of sub-systems is involved, because in this case, the system has always MP dynamics. This can greatly support the decision making process in the controller design problem.

Author Contributions: Methodology, A.M., R.F., and C.M.I.; software, A.M. and R.F.; validation, A.M., R.F., and C.M.I.; writing, original draft preparation, A.M. and R.F.; writing, review and editing, A.M. and C.M.I.; supervision C.M.I;

Funding: This research was funded by a Ghent University Special research fund Starting Grant "Minimal information model multi-objective predictive control for multivariable systems (MIMOPREC), no. 01N02018". RF was funded by the Erasmus Lifelong Learning Program for his exchange period at Ghent University, Belgium.

Conflicts of Interest: The authors declare no conflict of interest.

\section{Abbreviations}

LTI Linear Time Invariant

NMP Non-Minimum Phase

MP Minimum Phase

MIMO Multi-Input Multi-Output

LCM Least Common Multiple

GCD Greatest Common Divisor

RHP Right Half Plane

\section{Appendix A}

Proof of Proposition 4. Assume by contradiction: $\alpha_{0}>\beta_{0} \Rightarrow \alpha_{k}<\beta_{k} \quad \forall k=\overline{1, n}$.

If $T_{\alpha}=\min \{\mathcal{C}\}$ and $T_{\beta}=\max \{\mathcal{D}\}$, then:

$$
\begin{aligned}
& \alpha_{k}>\prod_{i=1}^{n} \gamma_{i} T_{\alpha}^{k} \frac{(2 n) !}{k !(2 n-k) !}=\hat{\alpha}_{k} \\
& \beta_{k}<\prod_{i=1}^{n}\left(1-\gamma_{i}\right) T_{\beta}^{k} \frac{n !}{k !(n-k) !}=\hat{\beta}_{k}
\end{aligned}
$$

resulting in $\alpha_{k}<\beta_{k} \Rightarrow \hat{\alpha}_{k}<\hat{\beta}_{k}$, i.e.,

$$
\prod_{i=1}^{n} \gamma_{i} T_{\alpha}^{k} \frac{(2 n) !}{k !(2 n-k) !}<\prod_{i=1}^{n}\left(1-\gamma_{i}\right) T_{\beta}^{k} \frac{n !}{k !(n-k) !}
$$


From the hypothesis, we have $\alpha_{0}>\beta_{0} \Rightarrow \prod_{i=1}^{n} \gamma_{i}>\prod_{i=1}^{n}\left(1-\gamma_{i}\right)$, then (A1) holds if and only if:

$$
\begin{aligned}
T_{\alpha}^{k} \frac{(2 n) !}{k !(2 n-k) !} & <T_{\beta}^{k} \frac{(n) !}{k !(n-k) !} \\
T_{\alpha}^{k} & <T_{\beta}^{k} \frac{n !}{k !(n-k) !} \frac{k !(2 n-k) !}{(2 n) !} \\
T_{\alpha} & <T_{\beta} \sqrt[k]{\frac{n !}{(n-k) !} \frac{(2 n-k) !}{(2 n) !}} \\
T_{\alpha} & <T_{\beta} \underbrace{\sqrt[k]{\frac{(2 n-k)(2 n-k-1) \cdots(2 n-k-n+1)}{2 n(2 n-1) \cdots(2 n-n+1)}}}_{p}
\end{aligned}
$$

To study the last inequality, the limits of $p$ are analyzed. Thus, when $k \rightarrow 1, p$ becomes:

$$
\lim _{k \rightarrow 1} p=\frac{n}{2 n}=\frac{1}{2}
$$

and when $k \rightarrow n$, we have:

$$
\begin{aligned}
\lim _{k \rightarrow n} p & =\sqrt[n]{\frac{(2 n-n)(2 n-n-1) \cdots(2 n-n-n+1)}{2 n(2 n-1) \cdots(2 n-n+1)}}= \\
& =\sqrt[n]{\frac{n(n-1) \cdots 1}{2 n(2 n-1) \cdots(n+1)}}<\frac{1}{2} \quad \forall n=2,3, . .
\end{aligned}
$$

Since the second case is less restrictive then the first case, only $k \rightarrow 1$ will be analyzed, obtaining:

$$
T_{\alpha}<\frac{T_{\beta}}{2} \Leftrightarrow 2 T_{\alpha}<T_{\beta}
$$

However, from second premise given in Proposition 4, it results that the product of two elements from $\mathcal{C}$ cannot be lower than an element from $\mathcal{D}$.

Hence, our assumption $\alpha_{0}>\beta_{0} \Rightarrow \alpha_{k}<\beta_{k}$ is incorrect, resulting in $\alpha_{0}>\beta_{0} \Rightarrow \alpha_{k}>\beta_{k}$.

Proof of Proposition 5. Firstly, we show that the function is strictly increasing in $I=(0,1)$. Then, to prove the unique real solution is sufficient to check the different sign in the function, when $\hat{\gamma}$ tends to the interval bound, finally, the values of the real solution are computed through algebraic calculations. The first derivative of $\zeta_{0}(\hat{\gamma})$ is:

$$
\zeta_{0}^{\prime}(\hat{\gamma})=n \hat{\gamma}^{n-1}-n(\hat{\gamma}-1)^{n-1}
$$

Since the hypothesis implies that $n$ is an even number, it results that $\zeta_{0}^{\prime}(\hat{\gamma})>0 \forall \hat{\gamma} \in I$. Thus, $\zeta_{0}(\hat{\gamma})$ is strictly increasing in $I$.

In the second part, the limits of the function are verified:

$$
\begin{aligned}
& \lim _{\hat{\gamma} \rightarrow 0^{+}} \zeta_{0}(\hat{\gamma})=-1 \\
& \lim _{\hat{\gamma} \rightarrow 1^{-}} \zeta_{0}(\hat{\gamma})=1
\end{aligned}
$$

Thus, the function has different signs in the interval extremes and is monotonically increasing, resulting in that it has only one real solution independent of the value of $n$. 
In the last part of the proof, we show that the unique real solution is equal to $\hat{\gamma}=\frac{1}{2}$. Hence, a real number must be found, such that:

$$
\begin{aligned}
\hat{\gamma}^{n}-(\hat{\gamma}-1)^{n} & =0 \\
\hat{\gamma}^{n} & =(\hat{\gamma}-1)^{n} \quad \forall n=2,4, \cdots
\end{aligned}
$$

Since in the hypothesis, $n$ is assumed an even number and $\hat{\gamma} \in(0,1)$, taking the $n$th root in the above equation, we have:

$$
\begin{aligned}
& \hat{\gamma}=-\hat{\gamma}+1 \\
& \hat{\gamma}=\frac{1}{2}
\end{aligned}
$$

Furthermore, it can be proven that that other $n-1$ complex solutions have all the same real part.

\section{References}

1. García, P.; Albertos, P.; Hägglund, T. Control of unstable non-minimum-phase delayed systems. J. Process Control 1999, 16, 1099-1111. [CrossRef]

2. Wang, J.; Zhang, Y.; Wang, W.; Zhao, J. Optimal design of PI/PD controller for non-minimum phase system. Trans. Inst. Meas. Control 2006, 28, 27-35. [CrossRef]

3. Yu, C.; Zhang, C.; Xie, L. Blind identification of non-minimum phase ARMA systems. Automatica 2013, 49, 1846-1854. [CrossRef]

4. Kumar, M.A.; Reddy, B.A. Robust PID Controller Design for Non-Minimum Phase Systems Using Magnitude Optimum and Multiple Integration and Numerical Optimization Methods. Int. Res. J. Eng. Technol. 2017, 4, 1474-1479.

5. Chen, D.; Peng, H. Analysis of non-minimum phase behaviour of PEM fuel cell membrane humidification systems. In Proceedings of the 2005 American Control Conference, Portland, OR, USA, 8-10 June 2005; pp. 3853-3858. [CrossRef]

6. Yuan, Z.; Wang, H.; Chen, B.; Zhao, J. Operating zone segregation of chemical reaction systems based on stability and non-minimum phase behaviour analysis. Chem. Eng. J. 2009, 155, 304-311. [CrossRef]

7. Campestrini, L.; Eckhard, D.; Gevers, M.; Balzanella, A. Virtual reference feedback tuning for non-minimum phase plants. Automatica 2011, 47, 1778-1784. [CrossRef]

8. Maestre, J.M.; Muñoz de la Peña, D.; Camacho, E.F.; Alamo, T. Distributed model predictive control based on agent negotiation. J. Process Control 2011, 21, 685-697. [CrossRef]

9. Di Nardo, A.; Cavallo, A.; Di Natalle, M.; Greco, R.; Santonastaso, G.F. Dynamic control of water distribution system based on network partitioning. Procedia Eng. 2016, 154, 1275-1282. [CrossRef]

10. Wang, Y.; Cembrano, G.; Puig, V.; Urrea, M.; Romera, J.; Saporta, D.; Valero, J.G.; Quevedo, J. Optimal Management of Barcelona Water Distribution Network using Non-linear Model Predictive Control. IFAC-PapersOnLine 2017, 50, 5380-5385. [CrossRef]

11. Muresan, C.; Ionescu, C.; Dulf, E.; Rusu-Both, R.; Folea, S. Advantage of low-cost predictive control: Study case on a train of distillation columns. Chem. Eng. Technol. 2018, 41, 1936-1948. [CrossRef]

12. Zhao, S.; Maxim, A.; Liu, S.; De Keyser, R.; Ionescu, C. Effect of control horizon in model predictive control for steam/water loop in large scale ships. Processes 2018, 6, 265. [CrossRef]

13. Juchem, J.; Lefebvre, S.; Mac, T.; Ionescu, C. An analysis of dynamic lighting control in landscape offices. IFAC-PapersOnLine 2018, 51, 232-237. [CrossRef]

14. Maxim, A.; Copot, D.; De Keyser, R.; Ionescu, C. An industrially relevant formulation of a distributed model predictive control algorithm based on minimal process information. J. Process Control 2018, 68, 240-253. [CrossRef]

15. Ferracuti, R. Control Adaptation Strategy Morphism to changing Process Architecture. Master's Thesis, Ghent University, Ghent, Belgium, 2016. 
16. Bequette, B.W. Process Control: Modelling, Design and Simulation; Prentice Hall: Upper Saddle River, NJ, USA, 2003.

17. Skogestad, S.; Postlethwaite, I. Multivariable Feedback Control: Analysis and Design, 2nd ed.; Wiley: Hoboken, NJ, USA, 2005.

(c)

(C) 2019 by the authors. Licensee MDPI, Basel, Switzerland. This article is an open access article distributed under the terms and conditions of the Creative Commons Attribution (CC BY) license (http:/ / creativecommons.org/licenses/by/4.0/). 